\title{
Miositis orbitaria, una causa de oftalmoplejia dolorosa en pediatría
}

\author{
Orbital myositis, a cause of pediatric painful ophthalmoplegia
}

\author{
Luisa María Parra-Rodas ${ }^{\mathrm{a}}$, Elízabeth Parra-Rodas ${ }^{\mathrm{b}}$, María José Jiménez-Villegas ${ }^{\mathrm{a}}$, \\ Yulieth Cartagena-Agudeloc, Dagoberto Cabrera-Hemer ${ }^{\mathrm{a}, \mathrm{d}}$
}

\author{
aDepartamento de Pediatría, Facultad de Medicina, Universidad de Antioquia. Medellín, Colombia. \\ bFundación Universitaria San Martín. Sabaneta, Colombia. \\ 'Departamento de Neurología, Facultad de Medicina, Universidad de Antioquia. Medellín, Colombia. \\ ¿Sección de Neurología Infantil, Hospital Universitario San Vicente Fundación. Medellín, Colombia.
}

Recibido: 29 de octubre de 2019; Aceptado: 18 de junio de 2020

\section{¿Qué se sabe del tema que trata este estudio?}

La miositis orbitaria es un proceso inflamatorio idiopático, inusualmente se presenta en edad pediátrica. Causa dolor ocular agudo, diplopía, limitación de los movimientos oculares y por sus secuelas es una emergencia oftalmológica. Los esteroides sistémicos son el pilar del tratamiento.

\section{¿Qué aporta este estudio a lo ya conocido?}

En nuestra paciente el diagnóstico se logró mediante la clínica, el estudio de otras patologías y la resonancia magnética de órbitas, sin necesidad de biopsia. El tratamiento con esteroides sistémicos fue exitoso con remisión completa durante el seguimiento.

\section{Resumen}

La miositis orbitaria $(\mathrm{MO})$ es un proceso inflamatorio grave de etiología desconocida que compromete los músculos extraoculares. La presentación en edad pediátrica es rara y con frecuencia afecta a más de un individuo de una familia, lo que sugiere algún grado de predisposición genética. Objetivo: Describir un caso de miositis orbitaria de presentación en edad pediátrica, sus características clínicas, y la utilidad de la imagen por resonancia magnética para la confirmación del diagnóstico. Caso Clínico: Paciente femenina de 13 años que presenta cefalea aguda, dolor periorbitario derecho, exacerbado con los movimientos oculares y visión borrosa a quien se le realizaron estudios para miopatía tiroidea, enfermedades infecciosas, autoinmunidad y cáncer que fueron negativos. En la imagen por resonancia magnética se evidenció miositis del músculo recto medio derecho, sin evidencia de neuritis óptica. Recibió tratamiento con glucocorticoides sistémicos intravenosos seguido de esteroides orales con mejoría clínica completa. Conclusiones: La MO tiene etiología desconocida, y puede tener un curso maligno. Dada su presentación clínica inespecífica, el estudio diagnóstico diferencial debe ser amplio, y su estudio debe considerar realizar resonanacia magnética. El inicio temprano del tratamiento con esteroides evita el daño permanente de los músculos extraoculares.
Palabras clave: Miositis Orbitaria; Dolor Ocular; Diplopía; Oftalmoplejia 


\begin{abstract}
Orbital myositis (OM) is a serious inflammation of extraocular muscles with unknown etiology. Pediatric presentation is rare and often affects more than one individual in a family, suggesting a genetic predisposition. Objective: To describe a pediatric case of orbital myositis, its clinical characteristics, and the usefulness of MRI for confirming the diagnosis. Clinical Case: A 13-year-old female patient presenting with acute headache, right periorbital pain, exacerbated by eye movements, and blurred vision. We ruled out thyrotoxic myopathy, infectious diseases, autoimmunity, and malignancy. An MRI showed right medial rectus muscle myositis and no evidence of optic neuritis. She was treated with intravenous systemic glucocorticoids followed by oral steroids with complete clinical resolution. Conclusions: OM has unknown etiology and can present a malignant course. Due to its unspecific clinical presentation, a comprehensive differential diagnosis should be made and it should consider performing MRI. Early treatment avoids permanent damage of extraocular muscles.
\end{abstract}

Keywords:

Orbital Myositis;

Diplopia;

Eye Pain;

Ophthalmoplegia

\section{Introducción}

La miositis orbitaria (MO) es una condición rara. Inicialmente fue llamada pseudotumor inflamatorio orbitario, y fue descrita por primera vez en 1903 por Gleason, Busse y Hochheim ${ }^{1}$. Es un proceso inflamatorio primario de los músculos extraoculares, de etiología desconocida. La miositis puede presentase de manera aislada o estar asociada a inflamación de tejidos blandos periorbitarios con dacrioadenitis, compromiso de la grasa orbitaria y perineuritis del nervio óptico, aunque este último es un hallazgo infrecuente ${ }^{1}$. Se cree que es desencadenada por un proceso autoinmune que involucra tanto la inmunidad celular como la humoral, responsable de ocasionar una microangiopatía mediada por complemento ${ }^{2,3}$.

Con frecuencia afecta a más de un individuo de una familia, lo que sugiere algún grado de predisposición genética ${ }^{4}$. La MO comparte características clínicas con enfermedades infecciosas, autoinmunes y oncológi$\mathrm{cas}^{5}$. El compromiso es predominantemente unilateral y típicamente cursa con oftalmoplejia dolorosa de presentación aguda o subaguda, eritema palpebral, quemosis y proptosis. La diplopía y la diminución de la agudeza visual pueden variar de acuerdo a la extensión del compromiso anatómico y son consideradas urgencias oftalmológicas ${ }^{5,6}$.

El diagnóstico temprano se relaciona con una mejor respuesta a los esteroides sistémicos, que son el tratamiento de primera línea ${ }^{7}$. Sin embargo, pese al tratamiento oportuno, la mitad de los casos recurren o cursan con dependencia de esteroides y otros tratamientos como radioterapia, agentes inmunosupresores e inmunoglobulinas 8 .

El objetivo de esta publicación es describir un caso de miositis orbitaria de presentación en edad pediátrica, sus características clínicas y la utilidad de la imagen por resonancia magnética (RM) de órbitas para la confirmación del diagnóstico, teniendo en cuenta posibles diagnósticos diferenciales. Esta publicación cuenta con el consentimiento informado de los padres y aprobación del Comité de Ética institucional.

\section{Caso Clínico}

Paciente femenina de 13 años de edad, sin antecedentes de importancia. Se presentó en el Servicio de Urgencias refiriendo una semana de evolución de cefalea, dolor periorbitario derecho, exacerbado con los movimientos oculares y visión borrosa. Recibió tratamiento inicialmente con Naproxeno con lo que mejoró la cefalea, pero no los síntomas visuales.

Al examen neuro-oftalmológico se observó disminución de la agudeza visual (20/25 bilateral con corrección), pupilas simétricas de $3 \mathrm{~mm}$ reactivas a la luz, sin defecto pupilar aferente relativo. Se evidenció limitación en la abducción del ojo derecho y dolor ipsilateral con los movimientos oculares. En el fondo de ojo se observaron ambos discos ópticos rosados, de bordes definidos, sin edema de la capa de fibras, mácula sana y retina adherida. No tenía signos inflamatorios periorbitarios.

Fue evaluada en el Servicio de Neurología Pediátrica y Oftalmología y se ampliaron estudios. Se descartó que se tratara de una deficiencia de vitaminas, el hemograma fue normal, la enzima convertidora de angiotensina fue negativa, la hormona estimulante de tiroides (TSH), triyodotironina (T3), tetrayodotironina libre (T4L), anticuerpos anti-peroxidasa tiroidea (antiTPO), anti-tiroglobulina (anti-Tg) y anti-receptor de TSH (anti-TRABs), estuvieron dentro de los rangos normales o fueron negativos. Adicionalmente, se realizaron pruebas de autoinmunidad que incluyeron anticuerpos antinucleares (ANA), anticuerpos contra antígenos nucleares extractables (ENA), anticuerpos anticitoplasma de neutrófilos (ANCA), factor reumatoideo y anticuerpos IgG4, los cuales fueron todos negativos. 
La electroforesis de proteínas fue normal. También se descartó infección por toxoplasma, sífilis y el estudio de LCR resultó normal.

Se realizó una RM de órbitas simple y con Gadolinio (figura 1), observándose en la secuencia T2 un incremento en la señal y engrosamiento del músculo recto interno derecho (figura 1-a). En la secuencia T2 con supresión grasa se observó un engrosamiento e hiperintensidad del músculo recto interno sin compromiso de las inserciones (figura 1-b). La secuencia T1 contrastada con supresión grasa mostró un engrosamiento del recto interno con captación del medio de contraste a este nivel y estriación de la grasa intra y extra conal (figura 1-c), lo que sugiere compromiso inflamatorio muscular. No se observaron alteraciones en el nervio óptico, con lo que se descartó la sospecha inicial de neuritis óptica.

Con estos hallazgos se hizo diagnóstico de MO del recto medio y se definió como plan de tratamiento 3 días de metilprednisolona a dosis de $1 \mathrm{~g}$ cada 24 horas intravenosa (IV), seguido de 3 días de metilprednisolona $250 \mathrm{mg}$ día IV y prednisolona $50 \mathrm{mg}$ vía oral hasta completar 14 días de tratamiento. La paciente fue dada de alta al octavo día de hospitalización, sin presencia de dolor, sin limitación de los movimientos oculares, con mejoría de la agudeza visual y sin diplopía. Durante el seguimiento de un año no presentó recurrencia de los síntomas.
Figura 1. A. RM de órbitas. Señal T2coronal: se observa incremento en la señal de T2 y engrosamiento del músculo recto interno derecho (flecha blanca). B. RM de órbitas. T2-axial con supresión grasa: se observa engrosamiento e hiperintensidad del músculo recto interno derecho con inserciones conservadas (flecha blanca). C. RM de órbitas. T1-axial contrastada con supresión grasa: se observa engrosamiento del recto interno, captación del medio de contraste a este nivel y estriación de la grasa intra y extra conal (flecha).
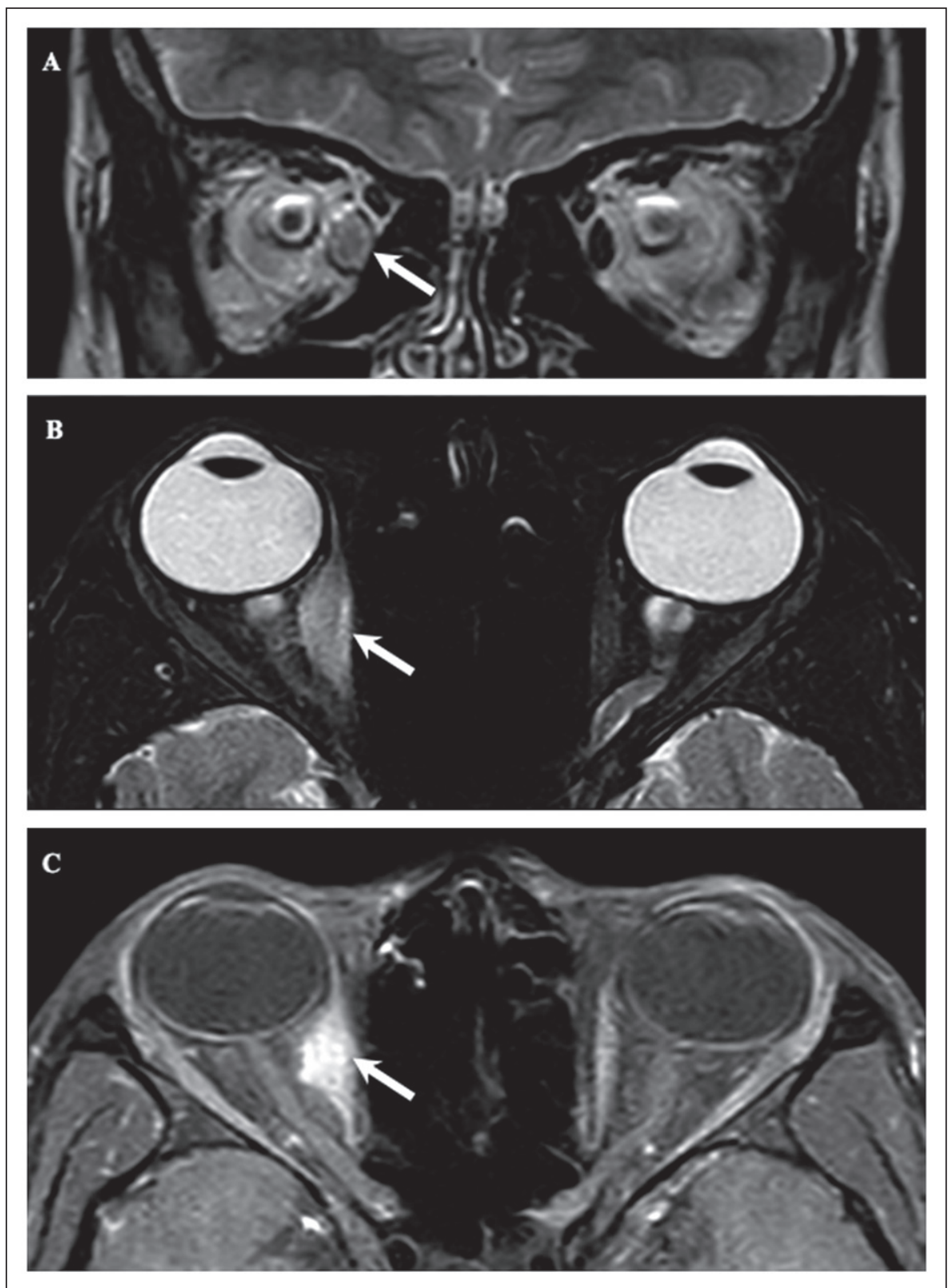


\section{Discusión}

La MO es una entidad rara en Pediatría, pues la edad de presentación más frecuente es entre la tercera y cuarta década de la vida ${ }^{7}$. Por esta razón los pacientes pediátricos requieren una evaluación clínica completa y un estudio riguroso de otras patologías con manifestaciones similares.

El espectro de presentación varía desde MO oligosintomática hasta un compromiso oculomotor grave ${ }^{5}$. En una serie de cinco casos con diagnóstico de MO, entre los 28 y 66 años, la diplopía y el dolor retrobulbar estuvieron presentes en los 5 pacientes, la inyección conjuntival en 2, los síntomas fueron binoculares en 2 pacientes y 3 tuvieron más de un músculo afectado. El músculo más frecuentemente comprometido fue el recto medio, demostrados por RM de órbitas 5 . El compromiso del músculo recto medial está descrito con mayor frecuencia que del recto inferior ${ }^{8} \mathrm{y}$ anecdóticamente se ha descrito compromiso del músculo elevador del párpado?.

El principal diagnóstico diferencial de la $\mathrm{MO}$ es la orbitopatía distiroidea que causa un engrosamiento de los músculos extraoculares, por lo que ambas condiciones se manifiestan con síntomas similares ${ }^{10}$. Otras etiologías que deben pensarse son la enfermedad de Lyme $^{11}$, infección por virus varicela Zoster ${ }^{12}$, Estreptococo del grupo $\mathrm{A}^{13} \mathrm{y}$ parásitos ${ }^{14}$, que usualmente se estudian según la epidemiología local. Las enfermedades autoinmunes como las vasculitis ${ }^{15}$, sarcoidosis ${ }^{16}$, lupus $^{17}$ y enfermedad de Crohn ${ }^{18}$ son otro grupo de posibles causas que se deben descartar. La enfermedad por anticuerpos IgG4 también cobra importancia dentro del diagnóstico diferencial, pues existen reportes de casos de MO por infiltración de células plasmáticas que expresan IgG4 ${ }^{19}$. Los autoanticuerpos séricos medidos en nuestra paciente para enfermedades reumatológicas fueron negativos, aunque para la enfermedad por IgG4 no se estudiaron anticuerpos contra el tejido ya que no se realizó estudio histopatológico por la mejoría con el tratamiento esteroidal.

Otra forma de orientar el estudio de los diagnósticos diferenciales es según el patrón de afectación mono o binocular. Por ejemplo, los tumores primarios o metastásicos, las malformaciones arteriovenosas ${ }^{5,6}$, la migraña oftalmopléjica y el síndrome de TolosaHunt usualmente son patologías monoculares, mientras que las enfermedades sistémicas autoinmunes, la oftalmopatía tiroidea, miastenia gravis ${ }^{14}$, distrofia muscular oculo-faríngea, síndrome de Kearns Sayre, el síndrome de Guillain-Barré variante Miller Fisher y las enfermedades mitocondriales tienen, por lo general compromiso binocular y suelen acompañarse de otras manifestaciones neurológicas 5 . En nuestra paciente el compromiso fue monoocular como en la mayoría de los reportes de $\mathrm{MO}$ y no se manifestó con otros signos neurológicos.

La RM de órbitas es el estudio imagenológico de elección ${ }^{5}$. Las secuencias de mayor utilidad son T2 y T1 contrastado con supresión de la grasa, Spin Echo y la secuencia Difusión. La apariencia típica de la MO es un engrosamiento de los músculos extraoculares afectados, aumento en la señal en T2 y captación del medio de contraste. A menudo se observa un realce con el contraste en la unión músculo tendinosa y la grasa circundante $^{20}$. Estas características la diferencian de la orbitopatía distiroidea que en la mayoría de los casos no compromete la unión músculo tendinosa, ni la grasa circundante, y usualmente respeta el recto lateral, y el músculo oblicuo superior ${ }^{10}$.

Los cambios imagenológicos en la $\mathrm{MO}$ pueden ser similares a los encontrados en la enfermedad relacionada con IgG4, pero esta se asocia a inflamación bilateral de las glándulas lacrimales y de los músculos extraoculares, especialmente el recto inferior, pero sin compromiso tendinoso ${ }^{19}$. En la fístula carotido-cavernosa pueden encontrarse signos de miositis acompañados por congestión venosa ${ }^{5}$, pero en esta condición las venas oftálmicas superiores se encuentran aumentadas de tamaño.

La presencia de metástasis o un linfoma pueden también confundirse con una miositis orbitaria, en las que se puede observar una masa focal con aumento de la intensidad de señal en los músculos extraocula$\mathrm{res}^{21}$. En la sarcoidosis, puede haber miositis pero rara vez es aislada y casi siempre se acompaña de uveítis e inflamación del seno cavernoso ${ }^{20}$.

El hallazgo histopatológico descrito en la $\mathrm{MO}$ es un infiltrado inespecífico de linfocitos, células plasmáticas e histiocitos. La biopsia es un procedimiento invasivo que expone al paciente a múltiples complicaciones por lo que se indica sólo en casos con inadecuada respuesta a los esteroides ${ }^{6}$.

El tratamiento de primera línea de la MO son los esteroides sistémicos ya que la mayoría de los pacientes mejoran rápidamente en los primeros días después del inicio del tratamiento ${ }^{22}$. El esteroide de elección es la prednisona oral, aunque también se ha usado, con buenos resultados la metilprednisolona intravenosa ${ }^{22}$. Otras terapias usadas son la combinación de betametasona intraorbitaria e indometacina ${ }^{23}$. Hasta el $60 \%$ de los pacientes que inicialmente no tienen buena respuesta a esteroides pueden recaer, en estos, se recomienda dar altas dosis de esteroides ${ }^{6}$.

En aquellos pacientes que recurren o que no responden a los esteroides deben iniciarse otro tipo de inmunosupresores. El más usado es el metotrexato ${ }^{24}$, aunque también se usan con cierta frecuencia la ciclosporina $\mathrm{A}$, ciclofosfamida y azatioprina ${ }^{5}$. Además, se ha probado eficacia en el tratamiento usando agentes 
biológicos, como el infliximab, que mostrado buen control de la enfermedad ${ }^{25}$. La elección del inmunosupresor debe individualizarse ya que no hay estudios concluyentes sobre cuál es el tratamiento con mayor eficacia $^{22-25}$. La radioterapia puede utilizarse si no hay respuesta al manejo inicial o si existe recurrencia ${ }^{5}$.

Usualmente el pronóstico es bueno siempre y cuando respondan al tratamiento ya que puede lograrse una completa recuperación de la función muscular. En los casos en los que menos se logra recuperación completa es en los que presentan recurrencia ${ }^{22}$.

\section{Conclusiones}

La MO es una enfermedad de etiología desconocida que puede tener un curso maligno. Dada su presentación clínica inespecífica, requiere un abordaje que permita descartar otras patologías que cursan con un cuadro clínico similar. Dentro del estudio es muy útil realizar una RM de órbitas, que permite evaluar de manera no invasiva todas las estructuras orbitarias. Conocer este diagnóstico es imprescindible para el estudio sistemático e inicio temprano del tratamiento con esteroides. El pronóstico depende de la respuesta al tratamiento, pero en la mayoría de los pacientes es bueno. La principal secuela es el compromiso grave de movimientos oculares y de la visión.

\section{Responsabilidades Éticas}

Protección de personas y animales: Los autores declaran que los procedimientos seguidos se conformaron a las normas éticas del comité de experimentación humana responsable y de acuerdo con la Asociación Médica Mundial y la Declaración de Helsinki.

Confidencialidad de los datos: Los autores declaran que han seguido los protocolos de su centro de trabajo sobre la publicación de datos de pacientes.

Derecho a la privacidad y consentimiento informado: Los autores han obtenido el consentimiento informado de los pacientes y/o sujetos referidos en el artículo. Este documento obra en poder del autor de correspondencia.

\section{Conflicto de intereses}

Los autores declaran no tener conflicto de intereses.

\section{Referencias}

1. Yuen SJ, Rubin PA. Idiopathic orbital inflammation: distribution, clinical features, and treatment outcome. Arch Ophthalmol. 2003;121(4):491-9.

2. Harris GJ. Idiopathic orbital inflammation: a pathogenetic construct and treatment strategy: The 2005 ASOPRS Foundation Lecture. Ophthalmic Plast Reconstr Surg. 2006;22(2):79-86.

3. Dalakas MC. Therapeutic targets in patients with inflammatory myopathies: present approaches and a look to the future. Neuromuscul Disord. 2006;16(4):223-36.

4. Jacob M, Ollagnon Roman E, Vighetto A. Familial orbital myositis. J Neurol. 2007;254(5):666-7.

5. Montagnese F, Wenninger S, Schoser B. "Orbiting around" the orbital myositis: clinical features, differential diagnosis and therapy. J Neurol. 2016;263(4):631-40.

6. Schoser BG. Ocular myositis: diagnostic assessment, differential diagnoses, and therapy of a rare muscle disease-five new cases and review. Clin Ophthalmol. 2007;1(1):37-42.

7. Yan J, Wu P. Idiopathic orbital myositis. J Craniofac Surg. 2014;25(3):884-7.

8. Gori S, Lucchesi C, Calabrese R, et al.
Inferior rectus myositis: a rare cause of painful ophthalmoplegia and a therapeutic challenge. Neurol Sci. 2013;34(5):789-91.

9. Almekhlafi MA, Fletcher WA. Levator palpebrae myositis. Neurology. 2008;71(15):1202.

10. Szczapa-Jagustyn J, Gotz-Więckowska A, Kocięcki J. An update on thyroidassociated ophthalmopathy in children and adolescents. J Pediatr Endocrinol Metab. 2016;29(10):1115-22.

11. Sauer A, Speeg-Schatz C, Hansmann Y. Two cases of orbital myositis as a rare feature of lyme borreliosis. Case Rep Infect Dis. 2011;2011:372470.

12. Patheja RS, Weaver T, Morris S. Unique case of orbital myositis and dacryoadenitis preceding the vesicular rash of herpes zoster ophthalmicus. Clin Exp Ophthalmol. 2016;44(2):138-40.

13. Alshaikh M, Kakakios AM, Kemp AS. Orbital myositis following streptococcal pharyngitis. J Paediatr Child Health. 2008;44(4):233-4.

14. Toshinobu Kubota (2011). Orbital Myositis, Idiopathic Inflammatory Myopathies-Recent Developments, Prof. Jan Tore Gran (Ed.), ISBN: 978-953-307694-2, InTech, Available from: http:// www.intechopen.com/books/idiopathicinflammatory-myopathies-recent- developments/orbital-myositis

15. Salam A, Meligonis G, Malhotra R. Superior oblique myositis as an early feature of orbital Wegener's granulomatosis. Orbit. 2008;27(3): 203-6.

16. Mavrikakis I, Rootman J. Diverse clinical presentations of orbital sarcoid. Am J Ophthalmol. 2007;144(5):769-75.

17. Arrico L, Abbouda A, Bianchi S, Malagola R. Acute monolateral proptosis and orbital myositis in a patient with discoid lupus erythematosus: a case report. J Med Case Rep. 2014;8:375.

18. Verma S, Kroeker KI, Fedorak RN. Adalimumab for orbital myositis in a patient with Crohn's disease who discontinued infliximab: a case report and review of the literature. BMC Gastroenterol. 2013;13:59.

19. Kim N, Yang HK, Kim JH, Hwang JM. IgG4-related ophthalmic disease involving extraocular muscles: case series. BMC Ophthalmol. 2018;18(1):162.

20. Pakdaman MN, Sepahdari AR, Elkhamary SM. Orbital inflammatory disease: Pictorial review and differential diagnosis. World J Radiol. 2014;6(4):10615.

21. Moon WJ, Na DG, Ryoo JW, et al. Orbital lymphoma and subacute or 
chronic inflammatory pseudotumor: differentiation with two-phase helical computed tomography. J Comput Assist Tomogr. 2003;27(4):510-6.

22. Ota M, Shimizu T, Yoshida H, Kawata A, Kinoshita M, Nakano S, Isozaki E, Matsubara S. (2015), Clinical features and therapeutic responses of idiopathic orbital myositis. Neurol Clin Neurosci 3:63-7.

23. El Nasser A Mohammad A. Local steroid injection for management of different types of acute idiopathic orbital inflammation: an 8-year study. Ophthalmic Plast Reconstr Surg. 2013;29(4):286-9.

24. Smith JR, Rosenbaum JT. A role for methotrexate in the management of non- infectious orbital inflammatory disease. $\mathrm{Br}$ J Ophthalmol. 2001;85(10):1220-4.

25. Garrity JA, Coleman AW, Matteson EL, Eggenberger ER, Waitzman DM. Treatment of recalcitrant idiopathic orbital inflammation (chronic orbital myositis) with infliximab. Am J Ophthalmol. 2004;138(6):925-30. 